\author{
Servicio de Asistencia Médico-Social \\ del Niñ̉o Reumático \\ de la Asistencia Pública. \\ "Hospital Pirovano".
}

Director: Prot. Dr. Joxé María Mäzera.

\title{
PROFILAXIS Y TRATAMIENTO DEL REUMATISMO (ENFERMEDAD DE BOULLAUD) EN LA INFANCIA
}

\author{
Por los Dres. Prof. Dr. JOSE MARIA MACERA, \\ ALBERTO PABLO RUCHELLI Y RODOLFO GAIG. \\ Baenos Aires. Argentina.
}

La profilaxis de la enfermedad reumática (enfermedad de Bouillaud) de la infancia, ha tomado vn amplio desarrollo en nuestro país en los últimos tiempos y ella está vinculada al inusitado incremento que esta enffermedad ha registrado en los últimos. Iustros; hecho que ha sido corroborado, por recientes estadísticas nacionales y extranjeras.

Desde el año 1932, iniciamos, junto con el Dr. Costa Bertani, desde el Hospital Salaberry, una campaña be profi. laxis contra esta enfermedad, cuyos resultados fueron presentados a la "Sociedad Argentina de Pediatria". en el año 1935. en una comunicación, titulada: "Cómo se ha encarado la lucha antirreumática infantil, en el Hospital Salaberry". Desde er.tonces, hemos continuado esta campaña en forma progresiva, la que actualmente se imparte desje el Hospital Pirovano $y$ en otros centros hospitalarios de la Capital Federal y del interior del país, de acuerdo a las normas y directivas que propusiéramos en aquella oportunidad. Son exponentes de esta campaña, nuestros estudios realizados sobre morbilidad infantil por cardiopatias reumáticas en el medio hospitalatio y escolar y asimismo. las estadisticas nacionales sobre mortálidad infantil por cardiopatias reumáticas.

Estas cifras y los numerosos casos de cardiopatías que a diario nos es dado observar, nos demuestra que todo lo he- 
cho hasta el presente, es aún poco, frente a lo mucho que puede realizarse, cuando los médicos y las autoridades sanitarías del país, se aboquen de una vez al problema de orden social, que esta grave enfermedad encierra. Para ello, se requiere darle al diagnóstico precoz y al tratamiento conveniente, la importancia que merece.

Corresponde, en este sentido, repetir las sabias frases cel gran maestro Morquio, cuando en 1935 escribía: "Consideramos que es ésta una de las cuestiones prácticas de la mayor importancia $\mathrm{y}$ donde la acción médica se mánifiesta con la más grande evidencia, favorable o desfavorable para el enfermo, según sea la atención que se le preste. Es uno de aquellos casos, en Pediatria, donke el médico puede hacer mucho bien o mucho mal; todo queda librado, a su ciencia y conciencia".

"Gran parte de los niños afectados de cardiopatias, no han recibido tratamiento oportuno, o lo han hecho en forma incompleta otras veces, por ignorar u olvidar, que puede esta enfermedad, desaparecer en su faz articular, pero continuar sv acción como manifestación cardíaca" (Morquio).

Después de citar a Morquio, la importancia social que debe atribuírsele a la profilaxis de esta enfermedad y lo mucho que puede esperarse de ella, al evitar la perniciosa acción que ejerce sobre la salud $y$ en el futuro del niño.

La profilaxis del Reumatismo de Bouillaud, es de orden médico y médico-social; ella debe ser planteada, desde luego. en tres fases.

La primera, se relaciona a la profilaxis de la enfermedad; 1. segunda, al tratamiento de la enfermedad, faz aguda $y$ convalecencia, es decir, profilaxis de la cardiopatía; y la tercera, a la asistencia social del niño cardíaco.

La primera faz, se basa, por excelencia, en una amplia campaña de difusión sobre las características clínicas tel mal, baciendo destacar especialmente. el peligro que significa esta enfermedad, por las secuelas cardíacas que puede determinar: en una palabra, se trataría de un plan de educación popular. citilizando para ello, los elementos más prácticos y de real utilidald: radio-difusión, cartillas, affiches, conferencias, avisos murales, etc., etc.

Entendemos que en esto estriba, fundamentalmente, la profilaxis de esta grave enfermedad; de modo que cuando el pueblo conozca. en debida forma los peligros a que estín expuestos los niños que suifren de dolores articulates, precozmente, de por si prestarán la atención que corresponda y harán tratar oportunamente, a los mismos. 
Esta es la obra que venimos cumplien'tio desde 1932, y Gue actualmente se imparte desde el Servicio de asistencia médico-social de la Asistencia Pública. cuya sade central está cn el Hospital Pirovano.

En las recientes Jornadas de Asistencia Sociai al Carcíaco, realizadas en Buenos Aires, en Abril del corriante año, bemos documentado esta acción en el trabajo expuesto sobre: "Plan de lucha antirreumática infantil, adoptitio por nuestro Servicio".

Consideramos asimismo que la profilaxis de esta gáave cnfermedad debe también desarrollarse en la escuela, el maestro, puedt ser un eficaz colaborador en esta campaña. El Cuerpo Médico Escolar actuaria despistando las cardiopatias y vigilando a tołlo niño que acuse dolores articularzs.

En el estudio sobre: "Las cardiopatías en nuestro medio escolar", sus proyecciones sociales: que realizamos en 1940 (estudio cardiológizo en 10,000 escolares), hemos encontradu que el Reumatismo articular rejvindicaba el $50,24 \%$ y que el Reumatismo cardíaco primitivo se encontró en el $0.64 \%$ del total de escolares examinados. Este estudio realizado con e: mayor rigorismo científico, reveló que el $2.1 \%$ de las carciopatias eran adquiridas y el $0,39 \%$ congénitas.

La segunda faz, de la Profilaxis, comprende la Profilaxis de las cardiopatías; ella depende casi exclusivamente de la acción đel médico, ya que está vinculada al tratamiento y va c'rigida a evitar en lo posible, las recidivas del mal. Se basará ella, en un conjunto te factores dignos de señalar:

1. Acción del reposo, en la prevención de las cardio. fatías. - Sin duda alguna, la clinoterapia es el tratamiento de mayor eficacia para prevenir o disminuir las localizaciones cardiacas que determina el Reumatismo de Bouillaud.

El reposo completo y prolongado en cama, ha sido adoptado por tolas las clínicas reumatológicas y nosotros la practicamos sistemáticamente en nuestro Servicio. con resultados halagüeños desde hace varios años.

Las investigaciones de Band y Duckett Jones (1939) sobre 1,000 niños teumáticos seguidos durante un período de 19 años. pone de relieve la bondad de este método. El $60 \%$ $c$ c los enfermos que han hecho clinoterapia severa y prolongada durante sus ataques reumáticos. después de 10 años de cbservación, llevan una vida normal. a pesar de que la mitad de ellos han hecho leves localizaciones cardíacas. En cambio. e! $40 \%$ de los restantes, que no han respetado la clinoterapia en la forma mencionada, el $25 \%$ de éstos fallecieron y el 
$15 \%$ restante llegaron a la edad addulta con moderadas o severas lesiones cardíacas y similar actividad física.

El tiempo que se debe mantener en cama a los niños reumáticos varía, de acuerdo a la intensidad de su Reumatismo y a la presencia o no, de localizaciones cardíacás. Este trempo cscila en nuestro Servicio, entre 2 a 6 meses.

Además de los síntomas de otden general, como ser: la fieblre, adelgazamiento, palidez, taquicardia, etc., etc., que indica actividad del virus reumático, y que son elemeritos de juicio para prolongar o no el reposo en cama, nosotros conrrolamos el tiempo de clinoterapia por la eritrosedimentación. prueba de laboratorio de la que no se debe prescinjür, si se desea seguir un tratamiento racional de estos entermos.

Mientras la velocidad de sedimentación de los glóbulos rojos esté muy acelerada, 13 indicación del reposo absoluto clebe ser exigida.

Entencemos por reposo absoluto, la inmovilidad estricta del niño en su lecho, no permitiéndole que lo abandone en ningún momento; el reposo debe ser en forma tal, que hasta la higiene personal y.las necesidades corporales, deberan ser hechas sin abandonar la cama; igualmente, se debe sxigir dentro de lo posible, el mayor reposo psíquico.

ruando el índice de Katz ha llegado a cifrąs normules. nosotros continuamos hacientio reposo por algún tiempo, aunque en forma ya moderada.

Si en el curso del Reumatismo aparece una carditis, se jebe ser más exigente con la clinoterapia, no menos de $3 \mathrm{me}$ ses. aunque la eritrosedimentación haya llegado a cifras normales.

Taussig y Goldemberg (1941) han demostrado, que el aumento progresivo del tamaño del corazón en los niños reumáticos en !desproporción al aumento que correspondería al crecimiento, evidencia una persistente infectión activa a nivel del corazón. Aun en las lesiones valvulares, este aumento progresivo, no se debe sino en parte mínima a la acción diriámica; la causa primordial es la lesión miocárdica. En estos casos, el reposo debe ser completo y estricto, a fin de colocar 2' corazón en condiciones de un mínimum de trabajo.

Estos autores han comprobado que el reposo puede evitar el aumento de volumen del corazón, aun en períodos de actividad reumática.

Esta disciplina es dificil mantenerla en nuestro ambiente, desde que el público no está compenetrado sobre la impor- 
tancia fundamental de estas medidas. Se resiste aceptarias y (i) permite el internaje, por períodos tan piolongados.

Cumplifa la internación en los casos de localización car diaca, en la forma descrita, la movilización posterior dzbera hacerse en forma progresiva, por etapas, con ejercicios graduaLios de marcha, etc. (tal como se lo practica en el hogar para niños convalecientes de Wickham), donde se contempla $I_{2}$ educación integral de los internados, de acuerdo a la capacidali fisica de cada enfermito.

Los resultados obtenidos con esta disciplina, son altamente favorables, desde que las estadisticas revelan quo lo.s niños que cumplen estrictamente con esta indicación. hacsi un 50 \% menos carbiopatías que los que no lo cumplen, como ya lo hemos mencionado (estadísticas de Band y Ducker fines).

2." Infecciones focales. - El concepto de la particivaiión de los focos sépticos, en especial, los amigdalinos y faxingeos, tiene amplia aceptación en Clínica Reumatológica ciesde hace muchos años.

Estudios experimentales de Mayer y las investigaciones de Davis, Curhman, Welhard y Munck, etc., y la de muchos ctros, especialmente $l_{a}$ de reúmatólogos re gran prestigio, y entre nosotros, las de Castex y su escuela, cstán contestes er la intervención de estos focos sépticos en la etiopatogenia de esta enfermedad.

Se ha llegado hasta creer. que los que presentan focos sépticos y no acusan reumatismo activo, son reumáticos en potencia y que bastaria la acción de un factor predisponent: o desencadenante, para que la enfermedad se exterioricz.

Para Lee, Mee y Worms, de acuerdo a sus investigaciones, niños operabos de sus amígdalas después de su primer ataque de Reumatismo, reducen sus recidivas a más del 90 \%: en cambio, los niños reumáticos operados tardiamente. sólo se benefician en un $20 \%$. Nuestra experiencia nos enseña, que existen casos de niños operados que han hecho repetidas t:. cidivas.

Kaiser investiga la acción de la ablación de amigdalas y vegetaciones adenoideas sobre la frecuencia cel Reumatismo. Establece que en 20,000 niños operados, la frecuencia del Reumatismo era menor en $1 / 3$. en relación a los niños no ope rados, que fueron 28.000, al mismo tiempo observó. que los niños operados y que hicieron posteriormente Reumatismo. acusaron un $50 \%$ menos cardiopatías que en los reumáticos no operados. 
Otros investigadores (Irigerman y Wilson), en cambio, encuentran escasa diferencia de recidivas a favot de los operados, $76 \%$ para los operados y $80 \%$ para los no operacos.

A pesar de la discrepancia con respecto a la frecuencia cie las recidivas reumáticas, la tendencia genetal es aceptar que se debe extirpar todo foco séptico (amigdalas y vegetaciones), siempte que el Reumatismo no esté en actividad clinica y hu;noral, concepto que aceptamos en un todo y que desde hace muchos años. to hemos puesto en práttica en nuestro Servicio en forma sistemática.

En cuanto a la oportunitad de la operación, recalcamos que sólo la ordenamos, toda vez que la eritrosedimentación liega a la normalidad.

3.' Acción del clima. - Está demostrado que el clima ásempeña un importante y real papel sobre la génesis, frecuencia y recidivas del mal.

E1 Reumatismo cardíaco es muy poco frectente en los países tropicales; en cambio se le observa en los paises fríos v húmedos, especialmente en zonas donde existe gran variación meteorológica. Intervienen con frecuencia en el primer episodio reumático, el enfriamiento y las mojaduras, siendo f:ctores predisponentes, la miseria, vida urbana, hacinamiento en viviendas mal ventiladas y húmedas, falta de abrigo. etc.; etc.

Señalamos, por ser ilustrativa, la experiencia obtenida vor Coburn en el año 1929. Este investigador, transportó a Puerto Rico. niños con cardiopatías teumáticas activas que estahan internados en Presbyteim (Hospital de New York) y que tenían sus amigdalas con pus: estos niños mejoraron en forma notable, a pesar de estar en un clima marítimo. Durante su estadía en Puerto Rico no registraron recidiva alguna, y estuvieron libres ije infecciones de las vías respiratorias superiores, pero al retornar a New York, muchos de ellos acusaron tecidivas del mal, precedidos, por lo general. de infecciones de las vías respiratorias superiores.

Esta experiencia demuestra, que el problema de las cardiopztías reumáticas en la infancia es un problema econó mico en gran parte, y que contando con Colonias di conva. Iecientes para niños reumáticos, en determinadas zonas del país, se logrará incalculables beneficios.

Parece que el contagio del Reumatismo, se produce cuatrdo aparece la angina inicial, la que habitualmente precede a las artralgias en 15 ó 20 días. 
Al respecto referimos las observaciones recogidas por Bernard Schlessinger (de Londres), en niños reumáticos, que al acusar una afección de la porción superior del aparato respiratorio (angina, por lo común) 15 ó 20 días después, presentaba recidivas del mal. Al suministrat aspirina en dosis altas a los mismos, mientras duraba la angina alrétedor de un mes, la frecuencia y severidad de las recaídas reumáticas que siguen a esas infecciones disminuyeron en forma ostensible; de ahí, entonces. la conveniencia de procuiar aislar en las Salas de niños a todo reumático que presente una angina y dar a éstos y a los niños que estuvieron en contacto con aquél'os aspirina o salicilato, en dosis convenientes.

4." Higiene personal. - Como esta enfermedad prevalece en las clases menesterosas, es necesario procurar evitar la habitación malsana, la falta de abrigos, la mala alimentación, etc., etc.

Todos estos factores puetlen eliminarse, internando a esta clase de niños, o de lo contrario, haciendo intervenir al Servicio Social, el que desde luego, debe contar con todos los elementos indispensables, y aplicarlos con la amplitud necesaria para conjurat los factores negativos mencionados, que pesan en la evolución de este mal.

5." Vacunación profiláctica. -.. Wilson y Swift, utilizan la vacunoterapia antiestreptocóccica, en el otoño e invietno y observan menos recaídas, que en los no vacunados.

Según Russell L. Cecil (de New York), si se desea obtener mejores resultados. conviene revacunar a los niños reumáticos cada año; de este modo, cree protegerlos mejor, pues parece que a los dos años, la inmunidad se pierde.

Nosotros no tenemos experiencia al respecto; es nuestro propósito, iniciar esta investigación.

6." Acción de la quimioterapia. -- En la Universidad de John Hopkins (Thomas y sus colaboradores), trataban a los reumáticos agudos con altas dosis de sulfamidas, desde 1936 a 1940 . Suministraton 1,20 de sulfanilamida por día a 55 pacientes y a otros 77 le hicieron la terapéutica clásica corriente, como contraprueba.

Los 55 enfermos recibieton sin inter rupción esta mediaación durante 6 meses (Noviembre-Abril), se cumplieron las pesquisas de hemogramas, para vigilar la lettcopenia, que a veces produce este medicamento, a las que no le asignaton importancia, por ser moderada en algunos casos.

Como conclusión de índole práctica. se observó que los tratados con sulfanilamidas no presentaton ninguna teciliva 
de importancia, ni ninguna otra infección aguda en el transcurso de la administración del medicamento.

Solamente un enfermo, hizo recidiva reumatica fuera del periodo de administración del medicamento, y liesde el punto de vista de lá investigación bactetiológica presentaban menor santidad de estreptococos en la faringe, en relación a los enfermos del grupo que no recibieron sulfanilamidas.

Pot otra parte, en los enfermos de control, existió en ecte mismo lapso. 15 episodios serios de recidivas reumáticas; en dos de ellos Endocarditis lenta mortal, en uno fiebre reumática aguda mortal y el cuarto falleció por causa indetermirada.

Por otta parte, Coburn y More, que han utilizado la sulfanilamida en la profilaxis del Reumatismo Infantil. observan que de $26^{\prime}$ niños que recibieron una dosis de 2 grs. iriarios del medicamento, en forma constaute. durante meses. sólo 1 tuvo una recidiva manifiesta, con buena tolerancia para la medicación, y con la particularidad que si la culfanilamida se suministra una vez íniciada la infección estreptocóccica (angina), no evita la recidiva del mal.

Estas largas investigationes demuestran la acción profiláctica que tienen las sulfanilamidas, para evitar las recidivas, $y$ revelan ser un poderoso agente profiláctico, contra las cardiopatías reumáticas, muy especialmente sobre la endocarditis bacteriana o estreptococo viritans.

I.üchtman y Bierman en 1941, han obtenido an buen portentale de curaciones, con este medicamento, ya solo, o ccmbinado con hipertermia artificial, vacuna antitífica (dia(trmia y onda corta).

Sobre 200 casos tratados, obtuvieron 12 curaciones, es aecir. el $6 \%$.

Segunda faz. - Profilaxis de la cardiopatia: comprencie el tratamiento en su faz aguda y en su convalecencia.

Desce que a esta enfermedad se la considera como afec. ción crónica, con poussées que pueden ser agudas o sub-agudas ii tratamiento debe ser sistemático, con curas de asalto y curas de entretenimiento.

Entre los agentes quimioterápicos utilizados. se destazan jos compuestos salicílicos, de los cuales e! más utilizado es et sulicilato de sodio, ai que se le considera, actualmente. com " meior medicamento existente.

La acción del salicilato ¿e sodio es rápida y eficaz: actáa en ocasiones de diagnósticos dudoscs. como piedra de to- 
quc (en la denominada prueba salicilada), definiendo situaciones a veces difíciles.

La droga debe ser pura, la dosificación y el ritmo de suministro bien precisada; es inocua para el organismo, siempre çue se le asocie alcalinos (bicarbonato de sodio u óxido de magnesio), doble dosis para el primero, mitad del segundo con relación a la dosis del salicilato de sodio, y siempre qut los filtros renales estén en buen estalío.

La dosificación del salicilato deberá ser relacionada. de dcuerdo a la intensidad de las manifestaciones clinicas y humorüles, especialmente si hay indicios de localización cardíaca.

Como se trata de una enfermedad que en su comienzo no sa sabe el grat́o de actividad del vírus, y cuál será en définitiva la evolución del proceso en lo que respecta a localización cardíaca; somos de opinión que conviene colocar al niño en las mejcres condiciones posibles ante esta enfermedad; de ahí. que en este caso agudo inicial o de recidiva aguda, utilicemos y propiciemos un tratamiento standard. Cuando se esté en presencia de una forma muy ligera, con antralgias siscretas, sin modificaciones electrocardiográficas y telerradiográficas. ccin tonos cardíacos bien timbrados y con una eritrosedimentación muy poco elevada, bastatá para yugular el mal un tratamiento menos intenso, menor cantidad de salicilato de sodio, por lo común, $0,50 \mathrm{gr}$, por año de edad y por día.

El tratamiento debe ser precoz, intenso y prolongado, iasando por las tres etapas: tratamiento de ataque, tratamienic regresivo y tratamiento de protección utilizando simultáneamente la vía oral y endovenosa o intramuscular, o si se clesea, Ia vía rectal en sus primeras 48 ó 72 horas $y$ a continuación seguir con las vías mencionadas.

Ios intervalos del suministro del medicamento, en su iniciación será de cada 2 ó 3 horas durante el día y la nocbe: de ese modo. se mantendrá al organismo bajo la acción constante de la impregnación medicamentosa, desde que se sabe que este medicamento se elimina en su grado máximo por la orina alrededor de1 $63 \%$ entre las dos a tres horas de su ingestión.

El tratamiento standiard, que desde hace más de seis años utilizamos y cuyos resultados hacemos conocer en este trabajo, es el siguiente: desinfección del rino faringe, con goras gomenoladas y tópicos de azul de metileno al $3 \%$ dos veces diarias, durante 20 dias; reposo tiguroso en cama, salicilato de sodio a la dosis de $1 \mathrm{gr}$. por año de eltad y por dia. distribuídos en intervalos de 2 a 3 horas (día y nocha): in. yección de $1 \mathrm{gr}$. de salicilato de sodio por vía intramuscular. 
Si la clínica revela que el proceso es grave, en lugar de una inyección de $1 \mathrm{gr}$. hacemos dos. con intervalo de 12 hor:s. Esta desis, por vía oral e intramuscular, la mantenemos durante 10 dias; después de este plazo, continuamos el tratamiento por vía oral con la misma medicación, pero descen. Giendo progresivamente ce $1 \mathrm{gr}$. de salicilato de sodio cada 8 dias, y haciendo con intervalos de 10 días. 1 gre de salicilato de sodio intramuscular durante 2 dias consecutivos.

No hemos observado ningún caso de ácido cetosis, con este tratamiento. Esto concuerda con la experiencia ce Lutembacher, quien trata por año más de 120 reumáticos agucos, sin registrar accidentes graves de ácicio cetosis. Se acepta hoy dia que existe una fragilidad hepática previa en la génesis de la ácido-cetosis, cuando se suministra salicilato de sodio en altas dosis.

En el curso de este tratamiento se vigila: la eritrosediraentación, el pulso, la temperatura, el funcionamiento r $r$ rial, el régimen dietético apropiado y el estado cardíaco por la clínica la electrocardiografia y la telerradiografía. Es decir que durante semanas, está el enfermito vigilado y bajo la acción de altas dosis de salicilato de sodio, que en forma gradual se le limita. hasta que llega a ingerir 1 gr. por día durante los últimos ocho dias, terminando de este modo ;su cura, siempre que su estado lo permita. Esta cura continuará en su domicilio, vigilado desde el Consultorio Externo, donde por 1. común. se le somete durante meses, a la ingestión de 3 grs. diarios, curante 10 dias consecutivos cada nes, vigilando su * itrosedimentación cada mes y su estado cardíaco cada 15 días.

En algunos casos, desde el Consultorio Externo, hacemos curas de refuerzo, entendiendo por tal. hacer cada 15 dias. i gr. de salicilato ce sodio endovencso o intramusiular, dos días seguidos.

$E_{n}$ líneas generales, esta técnica es la que ponemos en iráctiéa y dura alrededor de 2 años o más tiempo, si presenla cardiopatías.

Este tratamiento puede variar en cada caso, por factores múltiples; intolerancia al salicilato de sodio, reagudización del mal, enfermedades intercurrentes, ete., etc., adaptándonos a las circunstancias de cada observación en particular.

En un comienzo, en el Hospital Salaberry, utilizábamos là via endovenosa. con buep resultado y perfecta tolerancia, recurriendo al polisalicilato en proporción de: $(0,33$.\% de $\mathrm{Na}$. Ca. Y K., en solución al. $5 \%$ ). 
Se aplicaton más de 800 inyecciones; sus resultados se hicieron conocer en las "Jornadas Reumatológicas del Hospita! Salaberry" (año 1935).

Actualmente, desde hace varios años, utilizamos la vía intramuscular, con igual resultatio, teniendo la ventaja de ser más simple y fácil de aplicar.

No hemos observado intolerancia a estas dosis de salicilato, $y$ en los escasos enfermos que la registraron, hemos utilizado para yugular esta situación. la insulinoterapia 10 unidades dos reces al día, asociada al suero giuconado y al Clucolin o Dextropur, con buen éxito.

Como mordiente del salicilato cuando éste no actuaba, hemos asociado la proteinoterapia (leche), con resultasio favorable.

Existen, desde luego, el azufre coloidal (solución de $\mathrm{H}_{1}$ posulfíto de sodio al $10 \%$ ), la autohemoterapia, peptona ai $10 \%$, metales coloidales, que pueden igualmente utilizarse en estas circunstancias.

La opoterapia tiroidea, actuaria cuando existe la denoninada ausencia de reacción tiroidea ćefensiva (Sergent), actyando ésta como mordiente de las formas arrastradas, por verdadera acción substitutiva.

Con respecto al salicilato de sodio suministrado por enieroclisis, consideramos debe practicárselo en los casos de Reumatismo agusio intenso, utilizando la solución isotónica al 23,20 o/oo, en dosis de I gr. por año de edad y por día y como se ha dicho, durante 2 a 3 días, pára continuar luego el suministro del salicilato de sodio, por vía oral. Es de aita eficacia, desde que la clínica y la eritrosetdimentación lo de. muestran; se explica esta acción, porque de este modo se efactúa una absorción completa. y por encee, se produce una total impregnación, que es lo oue se persigut.

Si bien en el adulto. es de f́ácil aplicación, en el niño tiene sus inconvenientes por la modalidad propia de la infancia; de ahí, que lo hayamos aplicado en limitados casos.

El Piramicón. lo hemos utilizado cuando existió intolerancia para el salicilato de sodio por vía oral, con buena aceptación de parte del enfermo y a la dosis de hasta 2 grs. al día.

Creemos que es útil, en casos de intoletancia por el salicilato de sodio. En algunos enfermos agudos iniciamos el tratamiento con esta droga, pero a las 48 horas tuvimos que recurrir al salicilato de sodio, pues las artralgias, fie- 
bre, etc., etc., no cedian con la rapidez que se obtiene con 2i salicilato de sodio; de ahí nuestro entusiasmo por esta droga, que la consideramos como la más eficaz, hasta tanto no surja otra más activa.

El Piramidón tiene el inconveniente, de que expone a veces al enfermo a la agranulocitosis.

Las sulfanilamidas, la hemos atilizado, asociada al salicilato de sodio, como coasugunte del tratamiento (alrededor de 2 a 3 grs. al día), sin observar beneficios ostensibles. razón por la cual la abandonamos.

Es nuestro propósito volver a atilizarla, para corroborar si actúa o no, como profiláctica de las cardiopatías, de acuerco a lo ya enunciado anteriormente.

Creemos oportuno insistir sobre el concepto de las et.cacias terapéuticas, que se le atribuía hasta hace poco, al salicilato de sodio u otro medicamento en la cura de lesiones valvulares, consideradas como orgánicas.

Los modernos conceptos. en Clínica Reumatológica, demuestran que existen lesiones valvulares intersticiales. sin lesión del endocardio suprayacente; el virus reumático, llega al corazón por vía coronaria, y no por la sangre que circula en sus cavidades, provocando. por lo tanto insuficiencias valvalates funcionales por lesión de miocarditis $y$ valvulitis int:rsticial.

Como estas carbitis reumáticas son reversibles. es decir, susceptibles de desaparecer espontáneamente por ser un proceso exudativo. se explica la razón de la errónea interpretación de creer curada una lesión valvular atribuida a una endocatditis que no ba sido tal.

Por el contrario, si realmente existe una endocarditis. ésta es de carácter irreversible. desde que se trata ce un proceso proliferativo que conduce a la fibrosis cicatricial.

Tercera faz: Asistencia social del niño teumático. - Esta $s$ halla vinculada a la arción de la Visitadora de Higiene Social. cuya eficacia está demostrada ampliamente. Para ello es indisponsable contar con un Servicio Social bien organizado, ef que debe disponer to todos les elementos, a fin de hacer liegar al niño reumático los beneficios ąe su situación taquiera.

El niño reumático con cardiopatía, al llegar a la puber. tad debe ser orientado profesionalmente de acuerdo a sus ap. titudes y a la capacidad física que su lesión cardíaca petmita. En nuestro gais hemos sido los primeros y únices en abor- 
dar el estudio de la orientación profesional en los niños cardiacos (Día Médico N." 20, año 1940). Posteriormente, henos ampliado este estudio, que fué presentado en las Jornacias de Asistencia Social al Cardiaco en Abril del corriente año (actas de dichas jornadas).

Los resultados obtenisos hasta el presente, son tan alentadores que demuestran. los beneficios incalculables de esta acción social.

Resultados cbtenides en nuestio Servicio con el tratamiento que heme? picpiciado.

Hemos separado 100 historias clínicas de niños reumáticos internados en nuestro Servicio en los últimos años.

De estos 100 niños, 65 ingresarun con cardiopatias $y$ $35 \sin$ cardiopatías.

82 niños tenían de 8 a 13 años de edá y 18 niños tenían de 4 a 8 años de edad.

La mortalidad registrada fué del $6 \%$ bobre el total de ies 100 niños

De los 35 niños que ingresaron sin cardiopatías, 2 niños bicieron posteriormente carbiopatias, es decir, el $5,7 \%$ \% 6 niñes desaparecieton. ignorándose su evolución: 27 niños continuarcon hasta el presente sin lesión cardiaca, siendo éstos observados durante el siguiente lapso:

\begin{tabular}{|c|c|c|c|c|}
\hline & enfermos & durante & & año \\
\hline & " & ., & $1 y$ & \\
\hline & $"$ & " & 2 & \\
\hline & " & " & 4 & \\
\hline & " & $"$ & 5 & \\
\hline
\end{tabular}

De los 35 niños, 10 hicieron carditis reumática en los primeros días de su internación, 5 de estos fueron dados de itta sin carditis (miocarditis y valvulitis reumática) y los otros 5 fueron retirados del Servicio con persistencia, la que desaparució posteriormente.

De estos 35 niños, 113 acusaron durante su evolución trastornos electrocardiográficos de importancia.

Estos resultados son elocuentes como consecuencia del tratamiento stanijard, propuesto por nosotros y creemos que debe ser tomado como un tratamiento de orientación, hasta ianio otros mejoren estos porcentajes. 